\title{
The implementation of newborn screening for spinal muscular atrophy: the Australian experience
}

\author{
Didu S. T. Kariyawasam, MA, MBBS ${ }^{1,2}$, Jacqueline S. Russell, BSc, MN², Veronica Wiley, $\mathrm{PhD}^{4,5}$, \\ Ian E. Alexander, MBBS, $\mathrm{PhD}^{6,7}$ and Michelle A. Farrar, MBBS, PhD ${ }^{1,2}$
}

Purpose: To evaluate the implementation of the first statewide newborn screening (NBS) program for spinal muscular atrophy (SMA) in Australia. Processes that hinder and support clinical development, translation, and sustainability of the first primary genetic screening program in Australia are appraised.

Methods: The study prospectively describes the course (timelines, health processes, and preliminary clinical outcomes) for SMA screen-positive newborns from 1 August 2018 to 31 July 2019 in New South Wales and Australian Capital Territory, Australia.

Results: In the first year of the program, 103,903 newborns were screened. Ten newborns screened positive for SMA. Genetic confirmation of SMA occurred in $9 / 10(90 \%)$ of infants. Clinical signs of SMA evolved in 4/9 (44\%) within 4 weeks of life, heralded by hypotonia and weakness initially recognized in the neck. Median time to implementing a care plan (including commencement of

\section{disease-modifying therapies) was 26.5 days (16-37 days)} from birth.

Conclusion: NBS is essential for early and equitable identification of patients with SMA. Expedient diagnosis and management are vital, as disease latency appears brief in some cases. NBS shows significant clinical utility to support early parental decision making, improve access to specialist neuromuscular expertise, and facilitate initiation of personalized therapeutic strategies.

Genetics in Medicine (2020) 22:557-565; https://doi.org/10.1038/s41436019-0673-0

Keywords: newborn screening; spinal muscular atrophy; presymptomatic; implementation; translation

\section{INTRODUCTION}

The landscape for management of patients with spinal muscular atrophy (SMA) has changed irrevocably since the advent of disease-modifying treatments, thus revolutionizing clinical practice. ${ }^{1}$ Early commencement of treatment appears to offer the best prognosis for survival, reduction in need for permanent ventilation, and progress in motor development in symptomatic patients. ${ }^{2-5}$ The greatest benefit across all these parameters has been observed in infants treated within the presymptomatic period. ${ }^{6,7}$

With the emergence of these novel therapeutics for SMA, all traditional criteria from Wilson and Junger's World Health Organization principles of early detection have been satisfied, ${ }^{8}$ prompting the recent successful nomination and inclusion of newborn screening for this condition to the Recommended Uniform Screening Panel in the United States. ${ }^{9}$ While conventional clinical diagnostic pathways universally demonstrate significant diagnostic delays, averaging five months for the most severe phenotype, SMA type $1,{ }^{10}$ newborn screening (NBS) for SMA in contrast supports a more timely diagnosis, facilitating the implementation of proactive care to maximize clinical outcomes for affected infants.

The genetics of SMA lends itself to population-based genetic screening, with the vast majority of affected individuals having homozygous deletion of exon 7 of the SMN1 gene, mapped to chromosome 5q13. ${ }^{11}$ Effective technology for genetic screening for SMA now exists. Two recent pilot studies demonstrate that polymerase chain reaction (PCR) testing for this commonly found gene deletion accurately diagnoses SMA from dried blood samples (DBS) in $95 \%$ of cases, with no false negatives. ${ }^{11-13}$

In advance of forthcoming broad implementation of NBS for SMA, evaluation of these programs in a real-world setting is critical to support and sustain their successful long-term implementation. The unique nature and clinical consequences of genetic screening in the neonatal period require evaluation within a health-care setting. While other NBS programs for SMA have focused on methodology and validity of laboratory-based genetic screening, ${ }^{12,13}$ our primary aim is to describe the clinical journey for screen-positive newborns.

\footnotetext{
${ }^{1}$ Department of Neurology, Sydney Children's Hospital, Randwick, Sydney, NSW, Australia; ${ }^{2}$ School of Women's and Children's Health, University of New South Wales Medicine, University of New South Wales, Sydney, NSW, Australia; ${ }^{3}$ Department of Genetics, Sydney Children's Hospital, Randwick, Sydney, NSW, Australia; ${ }^{4}$ NSW Newborn Screening Programme, Children's Hospital Westmead, Westmead, NSW, Australia; ${ }^{5}$ Faculty of Medicine and Health, University of Sydney, Sydney, NSW, Australia; ${ }^{6}$ Gene Therapy Research Unit, Children's Medical Research Institute, Faculty of Medicine and Health, University of Sydney and Sydney Children's Hospital Network, Sydney, NSW, Australia; ${ }^{7}$ Discipline of Child and Adolescent Health, Sydney Medical School, Faculty of Medicine and Health, University of Sydney, Sydney, NSW, Australia. Correspondence:

Didu S. T. Kariyawasam (didu.kariyawasam@health.nsw.gov.au)
} 
The secondary purpose is to highlight how these clinical outcomes shape the implementation of a statewide NBS program for SMA. As such, we identify the barriers to implementation of the first genetic newborn screening program in Australia and examine processes that have proven effective to overcome these obstacles. As SMA enters a new treatment era, we focus specifically on the construction of a NBS service delivery model to identify and refer patients, provide treatment, and undertake long-term postscreening surveillance.

\section{MATERIALS AND METHODS}

\section{Participants}

Individuals were identified through a statewide NBS pilot for SMA in New South Wales and Australian Capital Territory (NSW/ACT) Australia between 1 August 2018 and 31 July 2019. Our study prospectively included neonates and infants (0-12 months old) who screened positive for SMA through this program. Legal guardians/parents of screen-positive newborns gave written informed consent to participate in the study.

\section{Consent for newborn screening for SMA in NSW/ACT}

An established NBS program for a spectrum of congenital diseases has been underway in NSW/ACT since the 1960s, derived from DBS taken within 48-72 hours of birth. ${ }^{14}$ All children born in NSW/ACT are offered screening, unless parents actively opt out of the process (which occurs for less than $0.1 \%$ of babies). In line with current practice, parents were provided with a pamphlet, had discussion with clinical staff, and were referred to the NSW NBS Program website to obtain further information as requested. The website was adapted to include parent information sheets and video resources for neuromuscular conditions.

\section{Newborn screening methodology}

First-tier screening for SMA was performed by the NSW/ACT Newborn Screening Laboratory. All DBS were analyzed for homozygous deletion of exon 7 of the SMN1, utilizing the Perkin Elmer real-time PCR 4-plex assay on a 96-well format and Thermostats QuantStudioTM DX platform. As such, newborns with one allelic deletion of exon 7 on SMN1 gene (i.e., compound heterozygotes or carriers) were not captured by the screening program. The genetic analysis was multiplexed with T-cell receptor excision circle (TREC) and kappadeleting recombination excision circle (KREC) as markers for primary immunodeficiencies. Digital droplet PCR (ddPCR) was used to determine $S M N 2$ copy number, undertaken as a second-tier screening test in all those who were SMN1 deletion positive (QX200 Auto DG Digital Droplet PCR system). The assay positive sample performance was validated using DBS from 34 known SMA patients (SMN2 copy number 2-4) using digital PCR System (QX200 ddPCR, Life Science Group). The analytical performance of the digital quantitative PCR (qPCR) assay showed 100\% sensitivity, specificity, and precision (two independent analyses to ensure result validity). Diagnostic testing for National Association of
Testing Authority (NATA) accredited confirmation of genetic status involved corroboration of SMN1 deletion results from serum samples (using P060-B2 SMA Multiplex Ligation Dependent Probe Amplification Kit [MRC-Holland]) by NSW South Eastern Area Laboratory Services (SEALS) pathology laboratory. SMN2 copy number was confirmed by qPCR by the Victorian Clinical Genetics Service.

\section{Screening, diagnostic, and postscreening surveillance pathway for screen-positive infants}

Infants with SMN1 deletion positivity and $<4$ copies of SMN2 on first DBS were considered screen-positive (Fig. 1). Current Australian health regulations allow licensed and reimbursed nusinersen therapy for symptomatic individuals $(<18$ years of age and with symptom onset $<3$ years) only. As previous studies have attested, individuals with $S M N 1$ deletion and $\geq 4$ copies of SMN2 are more likely to have a long latent phase, presenting with symptomatic SMA in late childhood or as adults. ${ }^{15}$ Thus, these individuals were not reported by our research pilot screening program to avoid possible psychological harm caused by providing an early diagnosis, with no immediate option for therapeutic intervention within the current therapeutic landscape in Australia. The pediatrician named on the DBS card and an assigned neuromuscular specialist based at Sydney Children's Hospital Network (SCHN), NSW were contacted by the NBS laboratory to flag the screen-positive result. After communication and delegation of roles between the pediatrician and specialist, the family was contacted by the assigned clinician and advised (over the telephone) of a screen-positive result for a neuromuscular disorder. All screen-positive newborns were invited to attend a centralized specialist neuromuscular service based at SCHN, NSW. A second DBS to verify initial screen results and diagnostic blood tests were completed, alongside a neurological assessment. The family was supported by the neuromuscular specialist, a genetic counselor, and a social worker. Once diagnostic results were available the family was invited back to clinic to discuss the implication of diagnostic results and next steps for management. Multidisciplinary review and clinical follow-up were facilitated from this point. Due to the high probability of early disease onset, (as predicted by SMN2 copy number) and to support decision making, repeated clinical assessment and electrophysiological measures (compound muscle action potential and electromyography) were conducted by a pediatric neuromuscular specialist to detect early features of disease onset while treatment was being planned in presymptomatic neonates. Longitudinal functional motor assessments, validated for use in patients with SMA type 1 (Children's Hospital of Philadelphia Infant Test of Neuromuscular Disorders/CHOP-INTEND) ${ }^{16}$ were completed by one designated senior physiotherapist to augment examination findings. This scale was developed for infants with neuromuscular disease, encompassing a range of motor function scored from 0 to 64 . Regression of motor function was considered indicative of clinical disease onset. Conversely, an increase in CHOP-INTEND scores of $\geq 4$, (prespecified 


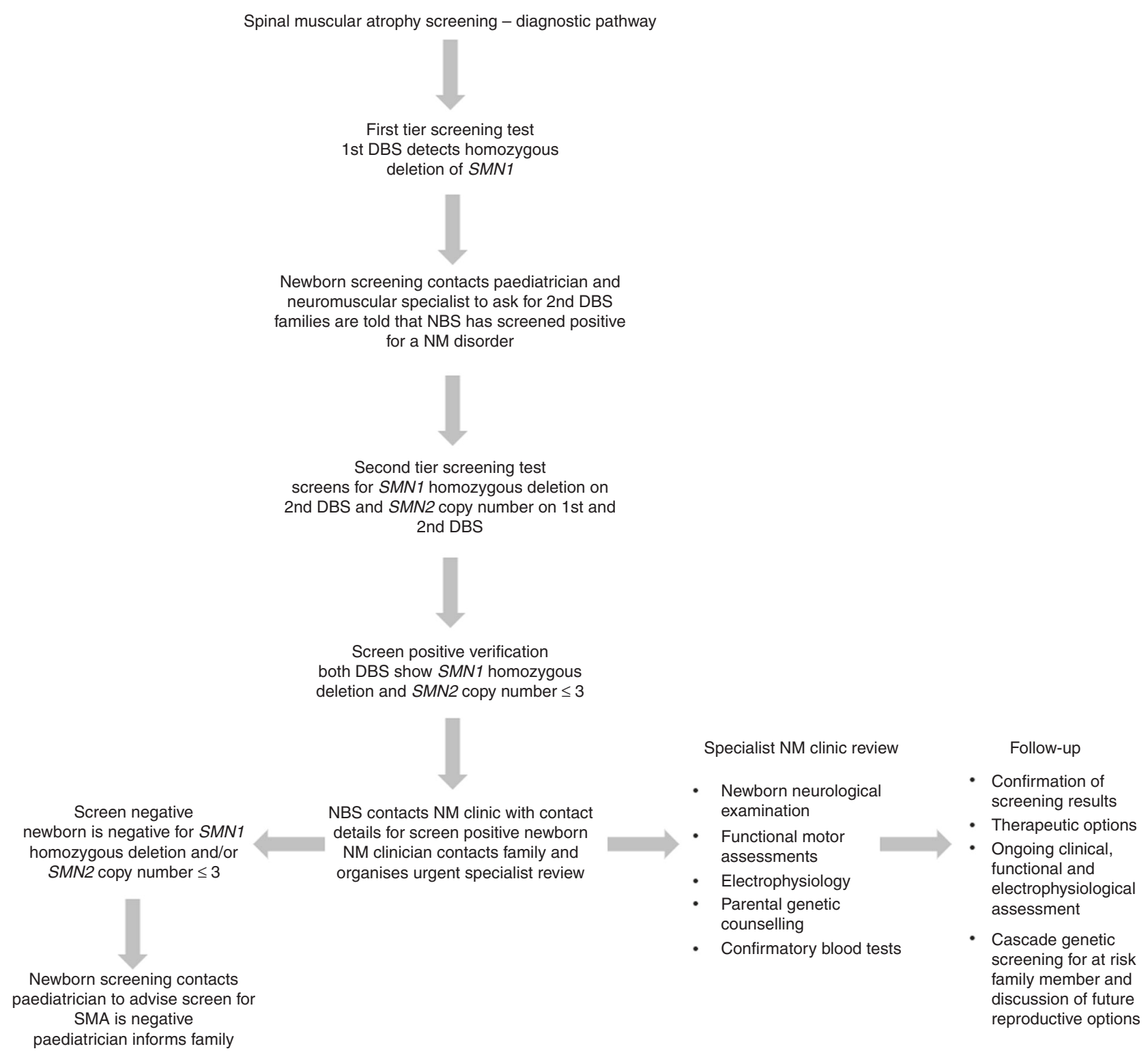

Fig. 1 The screening-diagnostic pathway for newborn screening in spinal muscular atrophy (SMA) in New South Wales/Australian Capital Territory. DBS dried blood samples, NM neuromuscular.

endpoint across clinical trials for SMA) was deemed a clinically significant improvement of motor function in our symptomatic cohort. ${ }^{2}$

Parents were counseled on genotype-phenotype correlation in SMA, allowing them to make informed decisions on management strategies for their newborn. Using data from previously published studies on SMN2 copy number (the most significant phenotypic modifier to date), they were counseled that untreated presymptomatic newborns with two SMN2 copies would have type 1 (would never sit) or 2 (would never walk) phenotype in approximately $82 \%$ and $17.2 \%$ of cases respectively. Untreated presymptomatic newborns with three SMN2 copies would have severe phenotypes (SMA type 1 and 2) in $17 \%$ and $70 \%$ of cases respectively. ${ }^{15}$ This guidance allowed our families to consider proactive intervention by being enrolled into a presymptomatic clinical trial (Rainbowfish [NCT03779334] or SPR1NT [NCT03505099]) or starting approved therapy on an individual compassionate basis. Alternatively, families were given the option of choosing a supportive model of care alone and/or starting diseasemodifying medication in response to symptom onset. Supportive care within a multidisciplinary setting, as dictated by SMA standard of care guidelines, continued to form the backbone of management for all infants. ${ }^{17,18}$

Methodology for adaptation of the NBS program for SMA The NBS program for SMA in NSW/ACT involved collaboration among multiple stakeholders across policy, diagnostic, and health-care systems. Methodologies that enhanced interdisciplinary learning, leading to multidisciplinary, patient-centered 
augmentation of care were essential in streamlining the program. ${ }^{19}$ The methodology for this study was adapted from Graham's Ottawa Model of Research, ${ }^{20}$ which has previously formed the backbone of World Health Organization-led frameworks for health care. ${ }^{21}$ This approach configures a sixstep knowledge translation model, guiding the process of transferring innovative research to effect deliberate and planned changes in clinical practice across multiple systems. ${ }^{20}$ As such, the timelines, clinical processes, and clinical outcomes for infants identified through the NBS program for SMA were reviewed. Study data were disseminated to relevant stakeholders in real time, through video conferences and statewide presentations, to hone clinical translation of the service.

\section{Ethics approval}

Ethics approval was gained from the Sydney Children's Hospital Network Human Research Ethics Committee prior to commencement of the study (HREC LNR/18/SCHN/307).

\section{Data analysis}

Data from the study were analyzed using descriptive statistics, utilizing frequency and percentage measures. Numerical data for timelines were represented by median values and full data range.

\section{RESULTS}

Demographic and genetic characteristics of the screenpositive cohort

From 1 August 2018 to 31 July 2019, 103,903 newborns were screened for SMA in NSW/ACT. Ten newborns were identified as screen-positive for homozygous SMN1 exon 7 and 8 deletions. Nine newborns were confirmed with this recessive deletion on diagnostic testing. However, on diagnostic testing one newborn with a consanguineous background was found to be a carrier for this variant (false positive on first and second DBS screening).

Of the diagnostically confirmed cohort, $6 / 9$ (67\%) had two SMN2 copies and 3/9 (33\%) had three SMN2 copies (Fig. 2). During genetic cascade screening $(n=8), 6 / 8(75 \%)$ couples had confirmed parental carrier status for exon 7 and/or 8 deletion in the SMN1 gene. In 2/8 (25\%) couples, paternal carrier status was not confirmed using standard multiplex ligation probe amplification. On further analysis, this was attributed in both cases to the presence of two copies of exon 7 and/or exon 8 on SMN1 on one allele, and deletion on the other (termed $2+0$ genotype).

Of our cohort, 6/10 (60\%) were males (Table 1). Families came from city-based areas in $7 / 10(70 \%)$ cases. Families identified as Australian in 4/10 (40 \%). In 4/10 (40\%) families with older children, no older sibling was symptomatic of SMA disease from parental history or neurological examination.

\section{Timelines for screening and diagnostic pathway for screen- positive newborns}

Median time to first DBS collection was 3 days from birth (range: $2-16$ days) (Table 2). In one newborn, DBS collection was delayed to day 16 of life due to a confounding medical complication. Initial DBS results were processed and available by 8 days of life (range: $5-18$ days) (Table 2 ). The median

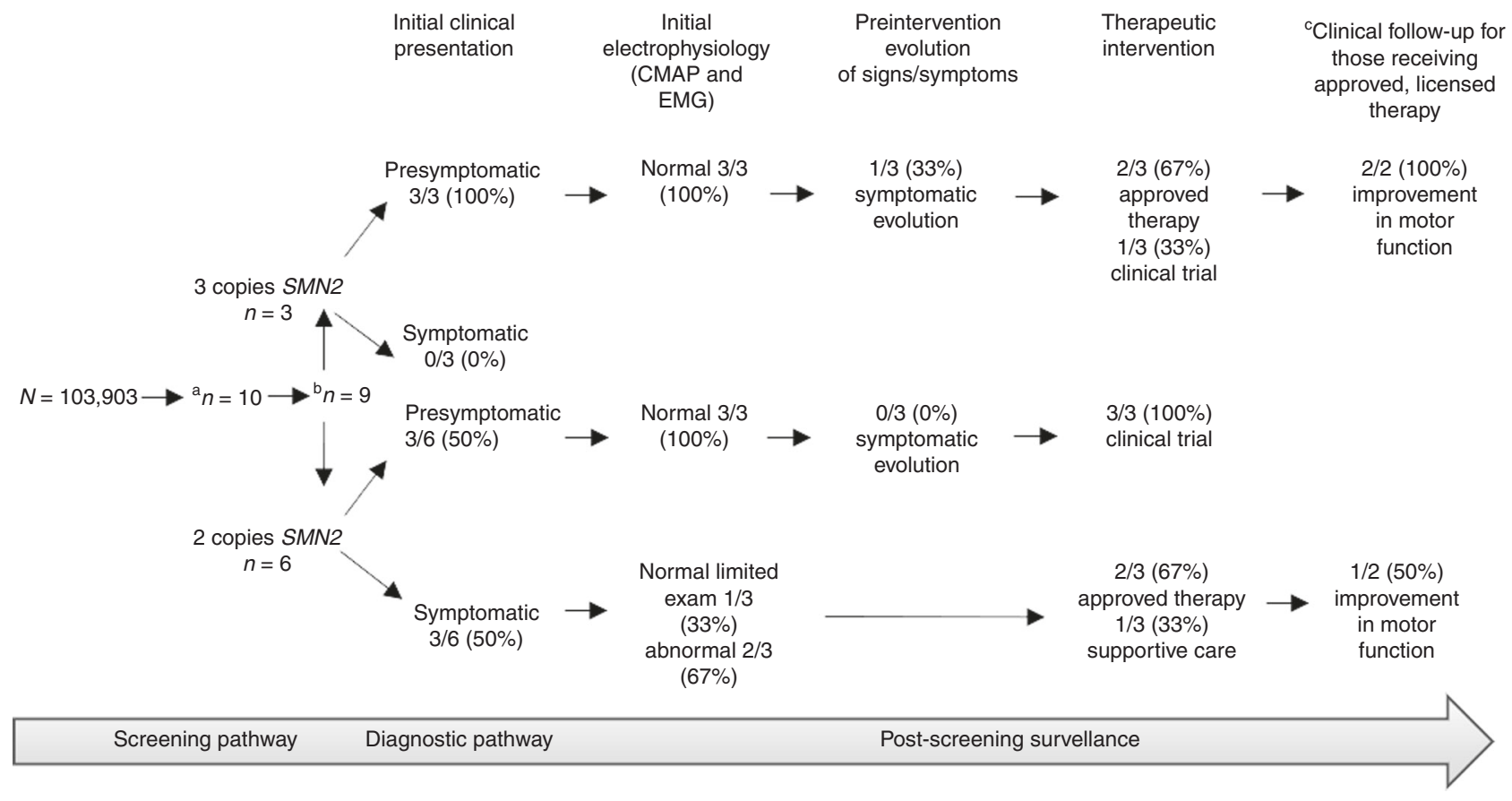

Fig. 2 The clinical pathway from screening to diagnosis and postsurveillance follow-up for screen-positive newborns. $N=$ Total number of

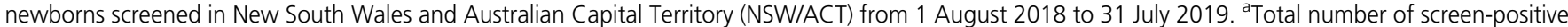

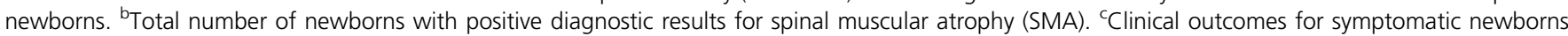
receiving approved and licensed disease-modifying therapy. CMAP compound muscle action potential, EMG electromyograph. 
time to specialist referral was 10.5 days of life (range: 5-18 days). Newborns identified as screen-positive were reviewed within the multidisciplinary setting of the neuromuscular service, at a median of 2.5 days from referral (range: 1-5 days). All newborns had diagnostic blood collected to validate initial screening results at the specialist review at 12.5 days of life (range: 8-23 days), with diagnostic results available at a median of 18.5 days (range: 13-24 days) of life. Thus, median time for completion of the screening-diagnostic pathway (from first DBS collection to availability of diagnostic results) was 13.5 days (range: 8-21 days). This allowed therapeutic planning and intervention to proceed by median of 26.5 days of life (range: 16-37 days).

\section{Clinical characteristics of diagnostically confirmed newborns}

Newborns $(n=9)$ who were diagnosed with SMA had a normal neurological examination as part of a mandated routine newborn check by a qualified pediatrician, within the first five days of life. None had signs consistent with the prenatal onset form of SMA (SMA type 0), clinically represented by decreased movements in utero, fetal akinesia deformation sequence (represented by joint contractures,

Table 1 Demographics of the screen-positive cohort and their parents, as identified through the NBS program for SMA

\begin{tabular}{lll} 
Demographic feature & Number $(n=10)$ & $\%$ \\
\hline Gender & 4 & 40 \\
\hline Females & 6 & 60 \\
\hline Males & & \\
\hline Parental country of origin & 4 & 40 \\
\hline Australia & 6 & 60 \\
\hline Overseas & & \\
\hline Parental primary language & 5 & 50 \\
\hline English & 5 & 50 \\
\hline Other & 6 & 75 \\
\hline Residence in Australia ${ }^{a}$ & 7 & 70 \\
\hline City & 3 & 30 \\
\hline Rural & & \\
\hline $\begin{array}{l}\text { Number of children } \\
\text { Screened child is first child }\end{array}$ & 6 & 60 \\
\hline Screened child has older siblings & 4 & 40 \\
\hline NBS newborn screening, SMA spinal muscular atrophy. \\
aGeographical area was defined using Australian Bureau of Statistics Geography \\
Standard (March 2018).
\end{tabular}

intrauterine growth restriction, and lung hypoplasia), cardiac anomalies, and quadriparesis. ${ }^{22}$ This severe form of SMA is associated with severe respiratory failure, bulbar difficulties at birth, and a universally fatal outcome by 1 month of life. ${ }^{22}$

Based on clinical and electrophysiological parameters of disease onset, $5 / 9$ (56\%) remained presymptomatic prior to therapeutic intervention (Fig. 2). In contrast, 4/9 (44\%) showed clinical signs consistent with SMA disease onset within the first 4 weeks of life. Of the latter subgroup, the majority $(n=3)$ had two copies of SMN2, while one newborn had three copies of this gene, evolving symptoms on day 33 of life, after an initial normal clinical assessment (Fig. 2). There was a spectrum of clinical presentation in symptomatic infants. Loss in head and neck strength and tone was observed as the earliest sign of disease in $4 / 4(100 \%)$ symptomatic infants, at a median of 20.5 days of life (range: 16-33 days). This was initially independent of other typical clinical features of SMA (absence of deep tendon reflexes, paucity of antigravity limb movements, absence of Galant reflex and absence of tongue fasicluations) in 3/4 (75\%) of the symptomatic cohort. In one newborn, however (case 9; Table 3), a typical constellation of signs of disease was noted on initial assessment on day 16 of life, including significant truncal and limb hypotonia and weakness, respiratory distress, absence of deep tendon reflexes and tongue fasiculation. Supporting these clinical features, electrophysiological measurements showed concomitant evidence of low compound muscle action potential (CMAP) in 2/4 (50\%) and denervated electromyograph (EMG) in 3/3 (100\%) (limited EMG was undertaken in one symptomatic neonate due to poor tolerance of procedure). Notably, electrophysiological studies demonstrated active denervation and normal CMAP in one neonate, preceding loss in head and neck strength and tone by five days.

\section{Clinical outcomes for diagnostically confirmed newborns}

The service delivery model in NSW/ACT included the postsurveillance care of newborns identified through the NBS program for SMA (Fig. 2). As of 31 July 2019, the followup period for the cohort ranged from 6 weeks to 12 months. Screen-positive newborns and their parents had access to genetic counselors, neurogeneticists, social workers, and members of the allied therapy teams. All newborns received concomitant supportive management through the expertise of the neuromuscular multidisciplinary team, per 2018 standards of care guidelines for SMA. ${ }^{18,23}$

There was universal parental agreement for early intervention in those who opted for proactive management.

Table 2 Temporal course for screen-positive newborns identified through the NBS program for SMA

\begin{tabular}{|c|c|c|c|c|c|c|}
\hline & 1st DBS collection & 1st DBS result & Referral to specialist clinic & Initial specialist review ${ }^{a}$ & Diagnostic blood result & Therapeutic intervention \\
\hline Number & 10 & 10 & 10 & 10 & 10 & 8 \\
\hline $\begin{array}{l}\text { Median (days) } \\
\text { Range (days) })^{b}\end{array}$ & $\begin{array}{l}3 \\
(2-16)\end{array}$ & $\begin{array}{l}8 \\
(5-18)\end{array}$ & $\begin{array}{l}10.5 \\
(5-18)\end{array}$ & $\begin{array}{l}12.5 \\
(8-23)\end{array}$ & $\begin{array}{l}18.5 \\
(13-24)\end{array}$ & $\begin{array}{l}26.5 \\
(16-37)\end{array}$ \\
\hline
\end{tabular}




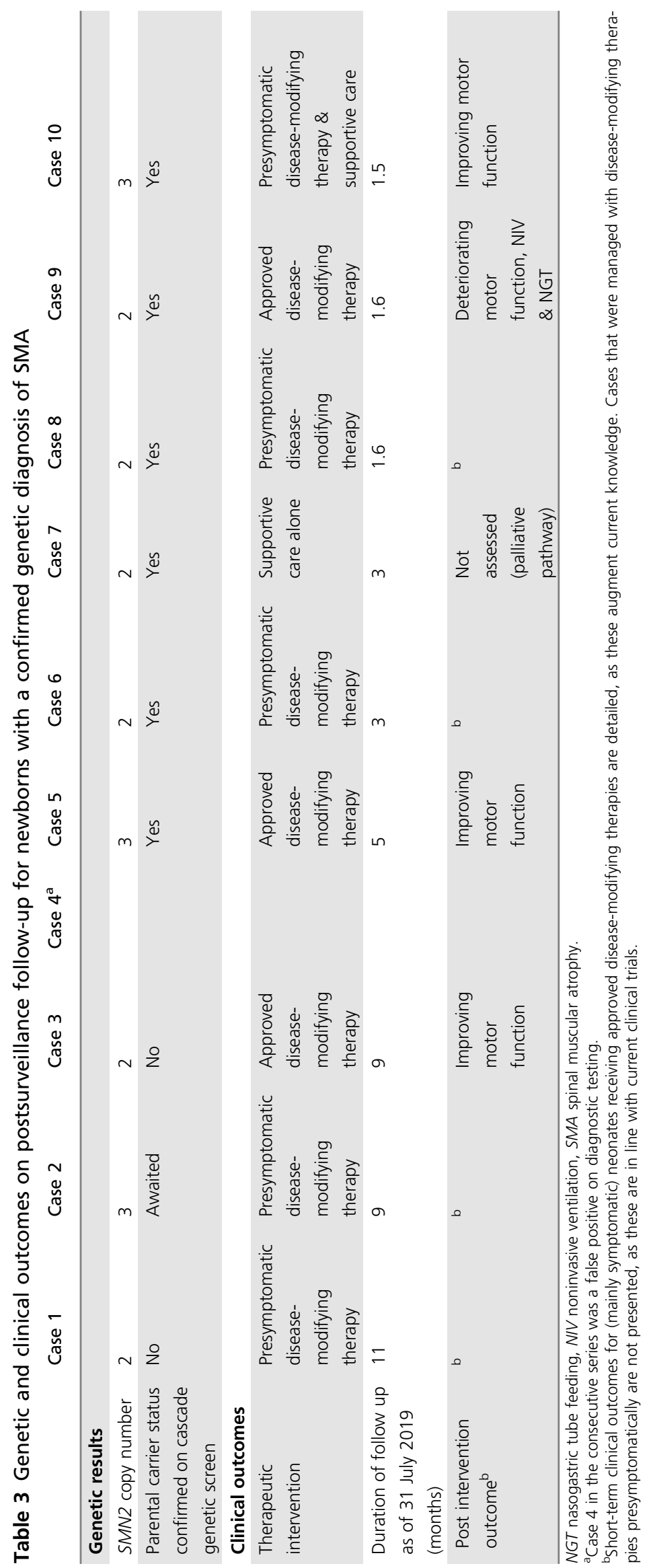


Accordingly, 8/9 (89\%) of the total cohort received some form of disease-modifying therapeutic intervention (Fig. 2). One family (case 7; Table 3) with an early-onset symptomatic newborn and additional serious medical comorbidities opted for a supportive pathway of care alone.

Infants who remained presymptomatic $(n=5)$ and were eligible enrolled into phase II/III clinical trials or requested individual compassionate access to approved and licensed therapy. In the symptomatic cohort, $3 / 4(75 \%)$ commenced treatment with intrathecal nusinersen combined with multidisciplinary supportive care (Fig. 2). Short-term monitoring of NBS outcomes has demonstrated clinically significant gains in motor function and milestones ( $\geq 4$-point increase in CHOP-INTEND scores) for $2 / 3$ (67\%) infants treated with nusinersen. Case 3, who started nusinersen therapy on day 21 of life, showed restoration of truncal tone, improvement in functional motor scores (CHOP-INTEND; baseline 37/64; age 4 months 58/64) (Table 3). Case 5 started nusinersen on day 33 of life. At age five months, neurological examination was normal aside from mild residual head lag on pull to sit (CHOP-INTEND baseline 59/64; age 5 months 61/64). Both patients remain free of permanent ventilation and are fully orally fed (Table 3). Case 9 showed the earliest and most significant signs of disease onset when first assessed on day 16 of life. Despite being treated with nusinersen expeditiously and at the earliest time point across the cohort, clinical progression occurred and by day 19 nocturnal noninvasive ventilation and nasogastric feeding commenced. Functional motor scores showed a clinically significant deterioration (CHOP-INTEND baseline 36/64, age one month 27/64) (Table 3).

\section{DISCUSSION}

Our pilot program represents the first primary genetic newborn screening program to be utilized in Australia. The epidemiological trends ${ }^{24}$ and false positive rate $^{13}$ identified through our study align with the global literature to date, confirming that this unique method of screening is accurate across the population to genetically detect SMA with high ascertainment. Despite preconception (carrier) screening for SMA being proposed as an alternative model to NBS, parental carrier status is difficult to determine in a proportion of couples, as seen in our study. Importantly, worldwide, $2 \%$ of carriers are compound heterozygotes ${ }^{25}$ and $6 \%$ carry two copies of exon 7 on SMN1 (including the aforementioned $2+$ 0 genotype). ${ }^{26}$ Thus, NBS programs for SMA remain essential for early diagnosis and treatment of SMA.

A key principle established in the Royal Australasian College of Physicians' Position Statement on Newborn Screening advocates equity of access to NBS programs in Australia. $^{27}$

Variabilities in access to health care and a spectrum of parental health literacy are acknowledged across the population of NSW/ACT ${ }^{28}$ and as such pose potential barriers to universal implementation and uptake of the service. Previous studies have concentrated on NBS programs for SMA in relatively small geographical catchment areas or homogeneous populations. ${ }^{12,13}$ In contrast, our study has established that NBS for SMA is feasible and reproducible throughout the vast geographical distances inherent to NSW/ACT and across the changing demographics of the Australian population. This has been facilitated by embedding genetic screening for SMA into already well-established and far-reaching NBS networks. Obstacles that may compromise the efficiency of the screening-diagnosis pathway include coordination of services that have not always been historically tightly interfaced such as screening/diagnostic laboratories and clinical institutions. As seen in our program, stakeholder engagement is vital to overcome such barriers to effective translation of the service. Pre-establishing defined roles and pathways for laboratory and clinical stakeholders allows smooth transition of the newborn and their family through the screening, diagnostic, and clinical surveillance process. Streamlining of the pathway is facilitated by utilizing key elements of the Ottawa model of research, employing a knowledge translation framework to create new, timely, and relevant knowledge, and link exchange efforts between these multiple health-care systems. ${ }^{20}$ Accordingly, international consensus opinions encouraging early identification of genetic status, and presymptomatic or early symptomatic intervention, can practicably be translated to a real-world setting. ${ }^{29}$

Previous studies have concentrated on the laboratory feasibility of NBS for SMA and contribute little to clinical aspects of screen-positive identification. ${ }^{12,13}$ Variability in clinical expertise among local pediatric health providers, especially for rare disease such as SMA, results in differences in practice, posing another potential barrier to effective postscreening support and surveillance of these newborns and their families. Our study, however, attests to the utility of integrating centralized postscreening surveillance into screening pathways to overcome such barriers. Channeling clinical and diagnostic expertise to defined specialist centers ensures that a universal and optimal standard of care is maintained through the clinical pipeline, expediting access to the tertiary neuromuscular care and multidisciplinary input required to manage this multisystem condition. Vitally, the implications of an early genetic diagnosis on the wider reproductive health of the family should not be underestimated. Early access to genetic counselors and social workers is crucial to support the far-reaching medical and psychological repercussions of this genetic diagnosis.

As seen in the preliminary clinical outcomes for our cohort, early identification of individuals through NBS allows multidisciplinary concomitant supportive care to be anticipated and implemented. This forms the backbone of standards of care for patients with SMA, reducing morbidities that are inherent to this diagnosis. ${ }^{30}$ Parents who opt out of treatment are equally supported by the expertise of the multidisciplinary team. Thus, early, informed parental decision making (independent of decisions to proceed with proactive management) is facilitated. As seen by the spectrum of clinical pathways taken by our diagnostically confirmed infants, NBS 
programs facilitate a personalized approach to management of infants with SMA. This patient-specific approach is vital within the current dynamic therapeutic landscape in SMA, where a standardized route of intervention in this early period is being established.

There is scant previous clinical data on the earliest characteristics of disease onset in SMA, leading to difficulties in targeting licensed therapeutic intervention to the earliest window of symptom manifestation. In contrast, our study provides a unique understanding of the clinical presentation of SMA identified through NBS. Onset may be early, characterized by a distinctive deterioration in neck strength and tone, particularly evident during dynamic assessment of truncal tone (pull to sit, supported sitting, and ventral suspension maneuvers). This sign may be initially independent of other typical features of SMA, including preservation of proximal antigravity movements and deep tendon reflexes. Previous large natural history studies have noted early manifestation of disease (within the first month of life) in only ten newborns cumulatively. ${ }^{30,31}$ In contrast, over $40 \%$ of our cohort evolved symptoms at first assessment or within weeks of birth, highlighting that disease onset may be early in a more significant proportion of infants than previously explored, with an often-precipitous progression in infants with $<4$ copies of SMN2. This is in keeping with the known swift, irreversible, and early motor neuron loss in the severest forms of disease, with up to $90 \%$ loss in the motor neuron pool at 3 months of age. ${ }^{31}$ The time-critical nature of identifying affected infants through NBS programs and having efficient processes to successfully confirm diagnosis is thus clinically emphasized by our study. Our findings show that in the real-word setting, clinical neurological assessment alone may not be sensitive enough to detect initial disease onset. Thus, other measures such as electrophysiology (CMAP and EMG) and longitudinal change in functional motor assessments are helpful as extensions to newborn examination. This aligns with international consensus opinions that recommend these adjuncts to improve sensitivity for detecting disease onset in untreated patients. ${ }^{29}$ Translating these research findings to clinical practice has optimized the effective delineation of presymptomatic and symptomatic individuals in our cohorts.

The short-term sequelae for our cohort demonstrate the significant clinical utility of NBS for SMA to provide access to early diagnosis and treatment, and subsequently improve clinical outcomes. Recently presented interim data from the relevant (ongoing, phase III) presymptomatic trial show that treatment improves survival, morbidity, and functional motor gains, in vast contrast to the natural history of untreated SMA. ${ }^{6,32}$ Similarly, in symptomatic patients receiving approved therapeutic intervention in our cohort, all were alive on follow-up as of the data cutoff period. The majority remained free of need for permanent assisted ventilation and supplemental feeding and showed clinically significant gains in motor skills over time. The clinical course of the earliest affected infant was supported by timely access to respiratory and feeding support, despite initial deterioration in functional motor skills during therapeutic induction.

The future of NBS for SMA in Australia centers on following longer-term outcomes for this unique group of newborns, to build real-world knowledge on their prognostic factors, counsel future families, and determine effectiveness of NBS for SMA in modifying clinical trajectories. This is especially important as the endpoints for tightly defined homogeneous clinical trial populations may not be truly reflective of the outcomes for the clinical spectrum of individuals captured through real-world NBS programs for SMA. Further studies for our own program will examine the acceptability of such programs within the populations they serve and also the cost-effectiveness of screening, early identification, and treatment to comprehensively understand the true ramifications of NBS for SMA.

\section{ACKNOWLEDGEMENTS}

D.S.T.K. acknowledges funding from the RTP Scholarship, University of New South Wales and the Freedman Family Foundation Scholarship. Validation of the multiplex qPCR and digital droplet PCR laboratory methods was supported by a Biogen Investigator Initiated Study. The NSW Pilot NBS study was funded by Paediatrio, a not for profit cooperative joint venture between Sydney Children's Hospitals Network, Children's Medical Research Institute, and Children's Cancer Institute established with the support of the NSW Government. Paediatrio is also affiliated with The University of Sydney and UNSW Sydney. The authors acknowledge Karen Herbert, Senior Neuromuscular Physiotherapist at Sydney Children's Hospital, who completed all functional motor assessments for the cohort.

\section{DISCLOSURE}

M.A.F. has received compensation as a member of the scientific advisory board for Biogen, Roche, and AveXis. D.S.T.K. has received reimbursement for attending symposia, sponsored by Biogen. These funding bodies had no role in the design of the study, data collection, data analysis, manuscript design, preparation of the manuscript, or decision to publish. V.W., I.E.A., and J.S.R. declare no conflicts of interest. The authors declare that the research was conducted in the absence of any commercial or financial relationships that could be construed as a potential conflict of interest.

Publisher's note Springer Nature remains neutral with regard to jurisdictional claims in published maps and institutional affiliations.

\section{REFERENCES}

1. Kariyawasam D, Carey KA, Jones KJ, Farrar MA. New and developing therapies in spinal muscular atrophy. Paediatr Respir Rev. 2018;28:3-10.

2. Finkel RS, Mercuri E, Darras BT, et al. Nusinersen versus sham control in infantile-onset spinal muscular atrophy. N Engl J Med. 2017;377: 1723-1732.

3. Lowes LP, Alfano LN, Arnold WD, et al. Impact of age and motor function in a phase $1 / 2 \mathrm{~A}$ study of infants with SMA type 1 receiving single-dose gene replacement therapy. Paediatr Neurol. 2019;98:39-45. 
4. Mercuri E, Darras BT, Chiriboga CA, et al. Nusinersen versus sham control in later-onset spinal muscular atrophy. $\mathrm{N}$ Engl J Med. 2018;378:625-635.

5. Messina S, Pane M, Sansone V, et al. Expanded access program with nusinersen in SMA type I in Italy: strengths and pitfalls of a successful experience. Neuromuscul Disord. 2017;27:1084-1086.

6. Bertini E, Hwu WL, Reyna SP, et al. Efficacy and safety of nusinersen in infants with presymptomatic spinal muscular atrophy (SMA): interim results from the NURTURE study. Eur J Paediatr Neurol. 2017;21:e14.

7. De Vivo DC, Topaloglu H, Swoboda KJ, et al. Nusinersen in infants who initiate treatment in a presymptomatic stage of spinal muscular atrophy (SMA): interim efficacy and safety results from the phase 2 NURTURE study (S25.001). Neurology. 2019;92(15 Suppl):S25.001.

8. Wilson J, Jungner $G$. Principles and practice of screening for disease. Geneva, WHO: Public Health Papers; 1968.

9. Kemper AR, Lam K, Comeau AM, et al. Evidence-based review of newborn screening for spinal muscular atrophy (SMA): final report (v5. 2). USA: Maternal Child Health Bureau, United States Secretary of Health and Human Services Advisory Committee on Heritable Disorders in Newborns and Children; 2018.

10. Farrar MA, Teoh HL, Carey KA, et al. Nusinersen for SMA: expanded access programme. J Neurol Neurosurg Psychiatry. 2018;89:937-942.

11. Lefebvre $S$, Burglen $L$, Reboullet $S$, et al. Identification and characterization of a spinal muscular atrophy-determining gene. Cell. 1995;80:155-165.

12. Kraszewski JN, Kay DM, Stevens CF, et al. Pilot study of population-based newborn screening for spinal muscular atrophy in New York State. Genet Med. 2018;20:608-613.

13. Chien YH, Chiang SC, Weng WC, et al. Presymptomatic diagnosis of spinal muscular atrophy through newborn screening. J Pediatr. 2017; 190:124. e121

14. Wilcken B, Wiley V. Fifty years of newborn screening. J Paediatr Child Health. 2015;51:103-107

15. Calucho M, Bernal S, Alias L, et al. Correlation between SMA type and SMN2 copy number revisited: an analysis of 625 unrelated Spanish patients and a compilation of 2834 reported cases. Neuromuscul Disord. 2018:28:208-215.

16. Glanzman AM, Mazzone E, Main M, et al. The Children's Hospital of Philadelphia Infant Test of Neuromuscular Disorders (CHOP INTEND): test development and reliability. Neuromuscul Disord. 2010;20:155-161.

17. Wang $\mathrm{CH}$, Finkel RS, Bertini ES, et al. Consensus statement for standard of care in spinal muscular atrophy. J Child Neurol. 2007;22:1027-1049.

18. Finkel RS, Mercuri $\mathrm{E}$, Meyer $\mathrm{OH}$, et al. Diagnosis and management of spinal muscular atrophy: Part 2: pulmonary and acute care; medications, supplements and immunizations; other organ systems; and ethics. Neuromuscul Disord. 2018;28:197-207.

19. Braithwaite J, Westbrook JI, Foxwell AR, et al. An action research protocol to strengthen system-wide inter-professional learning and practice [LP0775514]. BMC Health Serv Res. 2007;7:144-144.

20. Logan J, Graham ID. Toward a comprehensive interdisciplinary model of health care research use. Sci Commun. 1998;20:227-246.

21. Ellen ME, Panisset U, Araujo de Carvalho I, Goodwin J, Beard J. A knowledge translation framework on ageing and health. Health Policy. 2017;121:282-291.

22. Grotto S, Cuisset JM, Marret S, et al. Type 0 spinal muscular atrophy: further delineation of prenatal and postnatal features in 16 patients. J Neuromuscul Dis. 2016;3:487-495.

23. Mercuri E, Finkel RS, Muntoni F, et al. Diagnosis and management of spinal muscular atrophy: Part 1: recommendations for diagnosis, rehabilitation, orthopedic and nutritional care. Neuromuscul Disord. 2018;28:103-115.

24. Verhaart IEC, Robertson A, Wilson IJ, et al. Prevalence, incidence and carrier frequency of $5 q$-linked spinal muscular atrophy-a literature review. Orphanet J Rare Dis. 2017;12:124.

25. Prior TW, for the Professional Practice and Guidelines Committee. Carrier screening for spinal muscular atrophy. Genet Med.2008;10:840-842.

26. Smith M, Calabro V, Chong B, Gardiner N, Cowie S, du Sart D. Population screening and cascade testing for carriers of SMA. Eur J Hum Genet. 2007;15:759-766.

27. O'Leary P, Maxwell S. Newborn bloodspot screening policy framework for Australia. Australas Med J. 2015;8:292-298.

28. Mahimbo A, Seale $H$, Smith M, Heywood A. Challenges in immunisation service delivery for refugees in Australia: a health system perspective. Vaccine. 2017;35:5148-5155.

29. Glascock J, Sampson J, Haidet-Phillips A, et al. Treatment algorithm for infants diagnosed with spinal muscular atrophy through newborn screening. J Neuromuscul Dis. 2018;5:145-158.

30. Tizzano EF, Zafeiriou D. Prenatal aspects in spinal muscular atrophy: from early detection to early presymptomatic intervention. Eur J Paediatr Neurol. 2018;22:944-950.

31. Swoboda KJ, Prior TW, Scott CB, et al. Natural history of denervation in SMA: relation to age, SMN2 copy number, and function. Ann Neurol. 2005;57:704-712.

32. AveXis presented robust data at AAN demonstrating efficacy of Zolgensma $^{\circledR}$ in broad spectrum of spinal muscular atrophy (SMA) patients [press release]. 5 May 2019. https://www.novartis.com/news/ news-archive type $=$ press_release $\&$ page $=6$. 